\title{
GUIDELINES FOR SUBMISSIONS
}

Submissions, in English or French, should be sent to the following address:

The Editor

Theatre Research in Canada

c/o Graduate Centre for Drama, Theatre and Performance Studies

University of Toronto

2I4 College Street, $3^{\text {rd }}$ Floor

Toronto, ON

$\mathrm{M}_{5} \mathrm{~T}_{2} \mathrm{Z}_{9}$

Canada

FAX: I-4I6-97I-I378

Email: tric.rtac@utoronto.ca

Website: http://journals.hil.unb.ca/index.php/TRIC

Theatre Research in Canada / Recherches théâtrales au Canada welcomes submissions drawn from a full range of critical and historical approaches applied to the study of theatre as a multidisciplinary art, in the context of the cultures of Canada and Quebec. For our Forum Section we will consider position papers, commentary on issues of significance to the journal's mandate, information about new and old projects, and research notes. We will also consider the publication of fully annotated documentary evidence, normally appended to articles.

All submissions are refereed through a peer-review assessment process (please see the following Submissions Response Schedule). Full articles should normally be no longer than 7,000 words, typed double-spaced, following the internal editorial style found in the MLA Style Manual and Guide to Scholarly Publishing-3 ${ }^{\text {rd }}$ Edition (New York: Modern Language Association, 2008). Please submit articles, along with an abstract and brief biographical note, via email attachment (in Word for PC). Please employ an absolute minimum of document formatting in all electronic submissions (beyond the indentation of quotations and the use of endnotes). Endnotes are permitted (do not use footnotes), but should also be kept to a minimum. While TRIC/RTAC verifies all notes, references, and quotations, accuracy in these areas remains the responsibility of the contributor and any errors may delay publication significantly. All published articles will be included on our electronic website. 


\section{AVIS AUX CORRESPONDANTS}

Expédiez vos manuscrits en anglais ou en français à l'adresse suivante :

Rédacteur

Recherches théâtrales au Canada

Graduate Centre for Drama, Theatre and Performance Studies

Université de Toronto

2I4, rue College

Toronto, ON

$\mathrm{M}_{5} \mathrm{~T}_{2} \mathrm{Z}_{9}$

Canada

Télécopieur : I-4I6-97I-I378

Courrier électronique : tric.rtac@utoronto.ca

Site Web : http://journals.hil.unb.ca/index.php/TRIC

Recherches théâtrales au Canada / Theatre Research in Canada vous invite à proposer des articles inspirés de toutes les gammes d'approches critiques et historiques telles qu'elles s'appliquent à l'étude du théâtre en tant qu'art multidisciplinaire dans le contexte des cultures du Canada et du Québec. Pour notre Forum, nous prendrons en considération les exposés de principes, les observations sur des enjeux d'intérêt pour la revue, les informations sur des projets anciens et nouveaux et les notes de recherche. Nous pourrions également publier les notes bibliographiques annotées, normalement en annexe aux articles.

Tous les articles proposés sont arbitrés par des pairs (voir le calendrier des interventions pour des détails à ce sujet). Ils comptent normalement 7000 mots au plus et sont rédigés à double interligne suivant les règles stylistiques établies dans le MLA Style Manual and Guide to Scholarly Publishing-3 ${ }^{\text {rd }}$ Ed. (New York: Modern Language Association, 2008). Les auteurs sont priés de remettre leur article, accompagné d'un résumé et d'une brève notice biographique, en format électronique (Word pour PC). Pour la publication en format électronique, veuillez employer un minimum de formatage (à part la mise en retrait des citations et les notes de fin de document). Les notes de fin de document peuvent être employées (et non les notes de bas de page), mais nous demandons aux auteurs d'en limiter le nombre. Léquipe de $T R I C / R T A C$ vérifie l'exactitude des références et des citations, mais il incombe tout de même aux contributeurs de voir à leur exactitude. Toute erreur peut entraîner des délais de publication importants. Les articles publiés paraîtront sur notre site Web. 


\section{SUBMISSION RESPONSE SCHEDULE}

Theatre Research in Canada / Recherches Théâtrales au Canada will make every effort to process article submissions in a responsive, efficient manner. Under normal conditions, an author should receive an email acknowledgement from the journal within one week of receipt of a submission. Within the next four weeks the author should be informed as to whether or not the submission has been accepted for peer review. The journal anticipates that peer-review assessments will be completed and returned to our office within eight to twelve weeks. Therefore, under normal conditions, a submitting author should receive a summary of the review assessments no later than three to six months following the journal's receipt of the submission. If the journal experiences delays in the return of assessments, or if assessments need to be reassigned for any reason, the submitting author will be notified. Submitting authors who receive a positive assessment summary are requested to complete all necessary revisions and resubmit the finalized article within twelve additional weeks.

\section{CALENDRIER DES INTERVENTIONS}

Recherches théâtrales au Canada / Theatre Research in Canada s'assurera que les demandes de publication d'articles soient traitées de manière rapide et efficace. Normalement, l'auteur recevra un accusé de réception par courriel de la part de la rédaction dans la semaine suivant la réception de son article. Dans les quatre semaines suivantes, l'auteur devrait savoir si son article a été retenu pour l'évaluation par des pairs. Ces évaluations devraient par la suite être complétées et retournées à la rédaction dans les huit à douze semaines suivantes. Ainsi, dans des conditions normales, l'auteur qui propose un article devrait recevoir les résultats de l'évaluation dans les trois à six mois suivant la réception de son article. S'il y a des délais dans l'évaluation par les pairs, c'est-à-dire si les évaluateurs tardent à renvoyer leurs commentaires ou s'il faut pour une raison ou une autre confier l'évaluation à une autre personne, l'auteur en sera avisé. Advenant une évaluation positive, l'auteur devra apporter toutes les corrections nécessaires et remettre la version finale de son article dans les douze semaines suivantes. 